\title{
Bi-objective Assignment Model for Lean Order Picking in a Warehouse
}

\author{
Brigita GAJŠEK*, Hrvoje CAJNER, Marcin BUTLEWSKI, Tihomir OPETUK, Goran ĐUKIĆ
}

\begin{abstract}
With the introduction of Lean Warehousing, we are committed to using lean principles for more efficient warehousing operations, which are performed with quality and safety. Manual order picking, on which the paper is focused, is currently considered the most unfriendly to humans because, in the long run, it contributes to the appearance of musculoskeletal disorders. We record not only the increase in the average age of employees in warehouses but also in the number and duration of sick leave due to back and muscle pain. This paper explores the possibility of productive work while preventing order pickers from Work-Related Musculoskeletal Disorders. Using a laboratory experiment, we determine retrieval times for units with different characteristics and study required postures by guidelines of Revised NIOSH Lifting Equation. The final goal is to create a bi-objective assignment model.
\end{abstract}

Keywords: manual order picking; productivity; revised NIOSH lifting equation; warehousing; work-related musculoskeletal disorders

\section{INTRODUCTION}

Warehouses are always under pressure for more efficient, faster, and flawless performance. In order to achieve this, managers have recently been using Lean Warehousing. Lean is more common and widespread in manufacturing environments than in warehouses. Practice, in conjunction with Lean, often emphasizes raising productivity by eliminating wastes or non-value adding activities, also called Muda. In addition to Muda, Mura and Muri can also be found in the Toyota Production System. Unlike Muda, which focuses on wastes in processes, Mura and Muri are studying people and machines. In the case of Muri, if there is unevenness, then there are times where machines and people are overburdened [1]. The primary purpose of a just-in-time system is to eliminate the overburden of machines and people. Elimination of overburden could also be useful for order-pickers in warehouses, whose randomly assigned sets of tasks vary on the amounts of the total load. Mura refers to an unevenness in the distribution of workload in a system [1], for example, workshop or warehouse. A production process is a chain of linked and interdependent events. It is essential to ensure that every component of that process is tasked with a burden that they can handle [1]. In a situation of being overburdened, employees will lower their productivity or/and experience nausea, pain, and long-term sick leave.

The most labor-intensive and time-consuming activities in the warehouse are those from manual order picking [2, 3]. They account for more than 50\% of warehouse operating costs $[4,5]$. The process is itself an opportunity to improve the employees' productivity and reduce the number of circumstances that could lead to the occurrence of WRMDs in the long run. It differs from traditional production processes by the significant variability of tasks, which, in this case, are individual picks.

Manual order picking is the process in which workers are sent to products' storage locations to retrieve items for order fulfilment $[6,7]$. Today, many distribution entities are viewed as cost centres, not profit centres, and that becomes an obvious distinction on how managers approach order picking operations. Care for human health is often of secondary importance, as it is challenging to justify process automation. However, storage managers nevertheless suffer from extended and recurrent order pickers' sick leaves due to musculoskeletal disorders. Finding replacement workers due to the aging of the population is becoming increasingly difficult and time-consuming. Consequently, the concern for the health of employees is gaining importance, especially in terms of WRMDs prevention.

The cost per line ordered is the crucial measurement, which should be looked at daily. From this point of view, it is essential to know the distribution of an order picker's time, within which $50 \%$ of the time is spent on travel, $20 \%$ on searching, $15 \%$ on picking, and $10 \%$ on setup [5]. High pick density is desirable to decrease traveling time. A customer order that requires many picks per foot of aisle is relatively economical to retrieve because there is almost no cost for traveling. Still, it can add additional time for picking because of very likely stacking items along the vertical dimension of the rack. As far as traveling time is concerned, routing and storage policies influence mostly order picking costs and throughput times.

Searching times, as the second-largest time and cost item, can be decreased using technological solutions, like RF terminals, pick by light, pick by voice, pick by vision, and alike. The technology decision is mostly carried out at the warehouse planning stage and is not a matter of daily operational decisions. In contrast, picking times, as the third by overall duration, are also dependent on storage policies as confirmed by previous research.

For the same order, we can preserve order picking time at changed proportions of times for travel, search, and pick. We assume that viable alternatives differ in their contribution to the "daily dose" of safe collective demand of the job for order-picker. Incorporation of ergonomics justification in the determination of order picking scenario, in addition to traditional comparing picking times, could help to reduction in musculoskeletal disorders and the corresponding reduction in worker's compensation and other costs associated with these injuries. Manual order picking requires manual material handling tasks, such as lifting, lowering, and carrying, and is considered highly repetitive because the cycle times are usually 30 seconds or less. The cost of injuries and illnesses associated with manual material handling tasks is a significant factor for industry [8]. Occupational exposure to manual lifting and other ergonomic stressors has been associated with low back pain [9-12]. Low back pain affects roughly 40-80\% of the population at some point during their lifetime [13$15]$. 
Although Lean Warehousing is something new, it is based on a common platform with Lean Manufacturing, which is widely regarded as a potential methodology to improve productivity and decrease costs in manufacturing organisations. The lean concept is not just about increasing productivity but also about increasing safety. At order picking, safety also includes the prevention from WorkRelated Musculoskeletal Disorders (WRMDs) of order pickers, which should not be overloaded in an ergonomic sense. The success of lean manufacturing demands consistent and conscious efforts from the organisation [16]. This, however, is difficult to achieve in the case of Lean Warehousing in the field of order picking due to the diversity of tasks with different workload values, but not impossible, considering the contribution of industry 4.0. Research already proved the presence of a positive correlation between Lean manufacturing and Industry 4.0 [16]. IT, as an essential element of Industry 4.0, has the potential to support order-pickers with information about achieved daily workload, to propose ergonomically acceptable sequences of tasks, and more. All this makes sense to explore the possibility of a bi-objective assignment model, which will serve to high productivity at preserved Work-Related Musculoskeletal Disorders.

\section{LITERATURE REVIEW}

As the first who urged to incorporate human factors in order picking planning model, we recognised work from Grosse with co-authors [6], who reviewed more than 160 papers and suggested areas with research potential. One of them are bi-objective models combining productivity and ergonomics. That kind of models could help to design better order picking systems, friendly to owners and workers.

Grosse et al. [17] recognize two research papers, whose content overlaps with our efforts. Battini et al. [18] develop a bi-objective model that permits to compare and analyse the storage assignment solutions based on two functions: the first one considers the total order picking time, and the second one estimates the energy expenditure. Then, the rest allowance formulation of Price [19] is used to develop an integrated approach that considers both order picking time and energy consumed during the picking activities, where the latter permits us to consider ergonomic aspects in the model. Researchers' recommendations in [19] are valid for a specific orderpicking environment:

- a single-aisle;

- each item is stocked in a unique location;

- fixed and equally dimensioned storage locations;

- $\quad$ six different vertical storage levels $(0.25,0.58,0.94$, $1.30,1.63,1.96 \mathrm{~m})$;

- $\quad$ the depot (I/O point) at one end of the rack;

- $\quad$ a return routing policy;

- $\quad$ walking speed $1 \mathrm{~m} / \mathrm{s}$;

male operator: $75 \mathrm{~kg}$;

load of $0.2 \mathrm{~kg}$ per picked item.

As authors already discover, further analyses of the impact of orders profiles, especially the number of items (also mass) to be picked per line of an order, on the relative efficiency of different storage assignment policies are required. Larco et al. [20] research trade-off analysis between time-based function and ergonomics-based function. If Battini et al. [18] studied human factors through energy expenditure, Larco et al. [20] studied worker's discomfort using the Borg CR-10 scale [21], which is commonly used in the ergonomics field. Authors propose a unified methodology for order picking to quantify and balance two potentially conflicting criteria, namely (1) the short-term economic criterion of minimising total order-picking time, and (2) the human health criterion of minimising the incidence of WRMDs. Recommendations are similar to [18]. They are again tied to a particular warehouse environment:

- order-picking activities "pick-by-light" organised in zones with limited walking distances such that the retrieving times are an important component of the cycle time;

- $\quad$ picking products from totes on shelves at three levels (from 0.2 to $1.90 \mathrm{~m}$ );

- maximal mass of loads is $3 \mathrm{~kg}$;

- most picks involve single orderliness.

Data about the productivity aspect was retrieved from the Warehouse Management System. Additionally, workers who completed a questionnaire subjectively assessed the discomfort rate. Authors propose a simple heuristic that combines two criteria and popularity of a product. The product should be preferably stored close to depot and in the golden zone, whereby more frequent products are preferred for a more favourable location. Product stored in the golden zone allows effortless pick, without the need for bend over or raise hands above shoulders. Larco et al. [20] excluded the influence of mass from the model, which becomes less suitable for warehouses where heavier loads are manipulated. In their specific case, the results showed that volume is a better proxy than mass to handle complexities such as easiness to grab a product or easiness to retrieve a product from its location. The authors confirm that picking outside the golden zone does require additional retrieving time.

From the literature review presented above, we did not observe any attempt to design the bi-objective assignment model that would try to preserve order retrieval time and analyse a structure of required postures to minimize the incidence of WRMDs.

\section{METHODOLOGY}

Using a laboratory experiment, we determined retrieval times for different storage units placed on different heights in a warehouse rack. Time data was needed to address the productivity aspect. By observing the participants' movement, we studied required postures by guidelines of Revised NIOSH Lifting Equation (RNLE). Posture analysis was needed to address the WRMDs aspect.

Despite the fact that there are studies indicating additional risk factors for WRMD, such as body structure [27] or the differentiation of psychophysical abilities due to the age and sex of employees, these factors can be considered to a small extent as predictors of Musculoskeletal Disorders (MSDs) [28]. In the case of age, the difficulty of assessing the age itself is due to the selective nature of work (people who have suffered injuries stopped working in professions requiring specific effort) and the feeling of WRMDs symptoms against the 
background of general ailments occurring with age. Hence, in the applied Revised NIOSH Lifting Equation (RNLE), the individual features of subjects did not differentiate the results in terms of ergonomic risk.

\subsection{Laboratory Experiment and Time Study}

The laboratory experiment aimed to investigate the influence of the shelf height in the rack, item mass, and item dimensions on the required pick time. Pick time is a time that elapses between the moment the worker is located in front of the item's location in the rack in the upright position and the moment he/she defers an item that he/she previously took from its location in the rack. During this time, the worker does not move along the rack but performs work at a precise location along with the rack.

Participants were chosen to ensure the most representative sample that could be obtained. The composition of the group corresponds to the common age group of order pickers in practice. All participants were in good shape. Any of them could be hired as an order picker. With their help, we precisely determined the times of taking storage units from different heights. Researchers set these times by feeling and assume that the time of taking a storage unit increases proportionally with moving away from the golden zone up or down. The experiment was not intended to study WRMDs. Six men with a mean age of 32 (SD 9.3) years, the height of 181 (SD 3.9) $\mathrm{cm}$, weight of 86 (SD 11.7) $\mathrm{kg}$ and four women with a mean age of 30 (SD 9.7) years, the height of 166 (SD 9.6) $\mathrm{cm}$, the weight of 58 (SD 12.0) kg participated in the experiment. The participants were of different professions. Three men and two women were physical workers, one male and one female were students, and the rest were office workers. None of them has ever worked as an order picker in the warehouse or distribution centre. They all signed a consent form approved by the Senate of the Faculty of logistics at the University of Maribor.

The participants were picking cardboard boxes without handles in three different volumes and each volume with three different loadings from shelves on five different heights in a steel warehouse rack. We could also choose boxes with handles or some other shape, but we were guided in our selection by the fact that order picking is by far the most present in package distribution, where a box without handles is the most common object of handling. $80 \%$ of packages in package distribution weigh between a few decagrams and 10 kilograms. This led us to include both limit masses and the mass between them in the experiment, for the case of large and medium boxes. In small boxes, it was not possible to place the maximum mass of 10 kilograms inside them. For this reason, we chose different test masses for the small boxes. The maximum weight for small boxes was $5 \mathrm{~kg}$, and the minimum was the same as in the case of medium and large boxes. We took 1 kilogram of intermediate weight for small boxes, although one would expect 2.5 kilograms. One kilogram is much more common and distributed package. It comes in standard packaging and is the usual amount for personal consumption. Also, packages are in the distribution of packages of different sizes. We proceeded from a set of standard package dimensions and did not include boxes of exceptional, rarely present dimensions. On each height of the shelves we randomly stored 3 large $(L), 3$ medium $(M)$ and 3 small $(S)$ boxes, each size with three different loads $(L(0.1,5,10 \mathrm{~kg}), M$ $(0.1,5,10 \mathrm{~kg}), S(0.1,1,5 \mathrm{~kg})$ (Fig. 1). The dimensions of the large box were $31 \times 37 \times 45 \mathrm{~cm}$, the medium box measured $20 \times 30 \times 40 \mathrm{~cm}$, and a small one $7 \times 11 \times 18 \mathrm{~cm}$. Altogether, we stored 45 boxes numbered with Arabic numbers from 1 to 45 . The numerical designations served to identify the box that had to be picked.

Each participant was accurately informed about elements of the work environment (shelves, cardboard boxes, box masses, the desired motion of the body, a process). The need for rotating the entire body by $180^{\circ}$ without displacement from the front of the participant's body at the beginning or ending of the movement was specifically emphasized. Each participant performed a practice session of the work process until he/she felt relaxed, became ready to perform the required work, and stabilized his/her work pace.

During the experiment, each participant picked ordered box from a rack and lowered or lifted the box on an improvised belt conveyor behind him/her, using a grip of the box's side surfaces between the palms. The sequence of boxes on the picking list was determined randomly and was the same for all participants. After unloading the box on the conveyor, the assistant immediately read the new number of the box for picking. The lifting task retrieval starts when the participant's palms are placed on both sides of the required box, and the box starts moving from the shelve and ends when the box stops moving placed on the conveyor belt. The elapsed time between start- and end position of the box is defined as picking time.
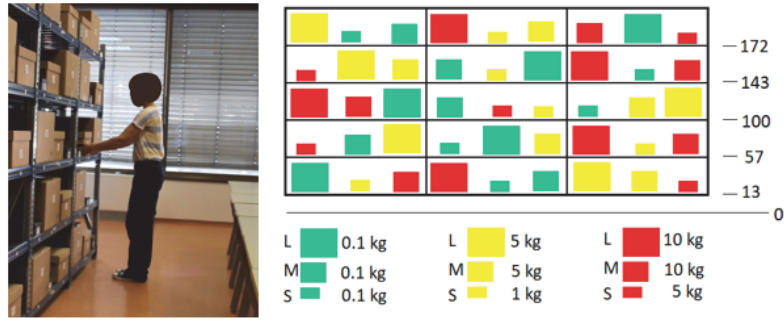

Figure 1 Laboratory environment with rack and improvised belt conveyor

A video camera with a rate of 60 frames per second recorded the participants. At the same time, they performed the tasks, and a time study was conducted by applying direct measurements on the video recordings. We investigated the influence of the box dimension, box mass, the initial height of the lifting/lowering (i.e., independent variables) on the picking time (i.e., dependent variables). The possible values of each of the independent variables are presented in Tab. 1.

\subsection{RNLE Procedure for Analysing Lifting Jobs}

For analysing lifting jobs, we used the Revised NIOSH Lifting Equation ( $R N L E)$, a tool for assessing two-handed manual lifting tasks. It permits the evaluation of asymmetrical manual lifting tasks and includes consideration of the quality of hand-container couplings and a large range of work durations and lifting frequencies.

$R N L E$ is a suitable tool for analysing in this paper researched picking tasks because: 
- manual handling activities other than lifting are minimal, account for less than $10 \%$ of the total worker activity;

- the environment is favourable (temperatures between $19{ }^{\circ} \mathrm{C}$ and $26^{\circ} \mathrm{C}$, humidity between $35 \%$ and $50 \%$ );

- tasks involve two-handed lifting, loads, and their centres of mass are stable.

Data collection was undertaken systematically according to the procedure proposed by [24]. Films were used throughout the evaluation, following the boxes from their origin to final destination. Measurements were taken with tape measures, meter sticks, electronic scale, and protractors. The analyst was trained in handling the devices employed in determining the variables at the worksite. The participants demonstrating the lifting tasks were instructed to perform the tasks consistently, so that accurate, repeatable measurements would be possible.

The collected data form the basis for the calculation of Recommended Weight Limit $(R W L)$ for a given manual lifting task. The $R N L E$ for calculating $R W L$ is based on a multiplicative model that provides the weighting for each of six task variables. The weights are expressed as coefficients that serve to decrease the load constant, which represents the maximum recommended load weight to be lifted under ideal conditions. The $R W L$ is obtained through Eq. (1).

$R W L=L C \times H M \times V M \times D M \times A M \times F M \times C M$

According to [22], $L C$ stands for Load constant, which is equal to $23 \mathrm{~kg}$. Horizontal Multiplier $(H M)$ is equal to $25 / H$ and is based on the horizontal distance from the ankles to the load $(H)$. Vertical Multiplier $(V M)$ is equal to $[1-(0.003 \cdot|V-75|)]$ and is based on the vertical position (height) of the load at the origin and destination $(V)$. Distance Multiplier $(D M)$ is equal to $[0.82+(4.5 / D)]$ and is based on the vertical distance through which the load is moved $(D)$. Asymmetric Multiplier $(A M)$ is equal to [1$(0.0032 A)]$ and represents Asymmetry Angle, the degree of twisting of the torso. Frequency Multiplier $(F M)$ is obtained from Tab. 5 in [22]. Coupling Multiplier $(C M)$ is obtained from Tab. 7 in [22]. $R W L$ values are then compared with the real weights of the objects lifted with the purpose of determining whether individual tasks need improvements to maintain the health of employees. The actual mass transferred by the employee, divided by $R W L$, allows to estimate the risk of manual handling tasks $(L I)$. Values above 3 indicate a high likelihood of lumbar spine injury $(L 4 / L 5)$. However, the risk may not have a linear course, which is emphasized by authors [26].

Nevertheless, the presented dependencies allow increasing the number of carried loads, their total mass during the work shift, thus increasing the productivity of the employee, with the indicator $(L I)$ unchanged. Therefore, better use of the employee's work can be made without exposing him/her to an increased risk of overloading. Modifications may also include working time as well as the number and length of breaks between consecutive manual material handling tasks.

In our experiment, weights and heights vary. In cases of such lifting, operations multitask analysis is used. Multitask analysis uses the single task $R W L$ equation and additional indexes to determine the overall cumulative or composite physical demands of the lifting station [23]. The Frequency Independent Recommended Weight Limit $(F I R W L)$ for each task reflects the compressive force and muscle strength required for a single repetition of that task [23]. The FIRWIL is obtained by multiplying $L C \times H M \times$ $V M \times D M \times A M \times C M$. The Single Task Recommended Weight Limit $(S T R W L)$ for each task reflects the overall demands of one task within the job and is calculated by multiplying the FIRWL $\times F M$ for each task [23]. The Frequency Independent Lifting Index (FILI) identifies potential strength problems for infrequent lifts and is obtained by dividing the load $(L)$ for each task by the respective FIRWL.

The Single Task Lifting Index (STLI) for each task is computed by dividing the average load weight $(L)$ for that task by the respective STRWL [23]. The STLI is used to identify individual tasks with excessive physical demands [23]. If any STLI value exceeds 1.0, then ergonomic changes may be needed to reduce the physical demands of the task.

The Composite Lifting Index (CLI) determines the additive effect of several tasks and is obtained by renumbering the tasks in decreasing order of physical difficulty as indicated by the STLI of each task [23]. The $C L I$ equation is listed in Fig. 2.

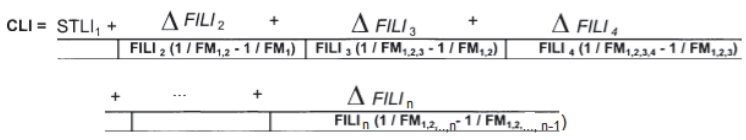

Figure 2 Step 3 of Multi-Task Job Analysis Worksheet [22]

The subscripts in the $C L I$ equation refer to the renumbered STLI sequence and are summed to determine a combined frequency multiplier $(F M)$ [23]. The $F$ (frequency rate) for the new renumbered tasks (subscripts of the equation) is summed for a total frequency rate too. The appropriate $F M$ is obtained from Frequency Multiplier Table in [22].

If the FILI of the first task is less than 1.0 then the first task is not stressful for the order-picker in terms of strength requirements. If any of the STLIs for the following tasks are greater than 1.0, that indicates a stressful situation, and the procedure should be corrected.

\section{RESULTS}

The factorial design was used as a basis for the experimental plan with factors, as shown in Tab. 1. The design was performed in randomized order avoiding the possible nuisance factors. One response variable was measured (picking time), and another one regarding the impact of lifting task on the risk of the worker's injury was calculated using previously explained RNLE. The calculation was performed using the input data from the experiment (design) and provided by the FILI index.

\subsection{Picking Time Model}

After analysis of the gathered data, the ANOVA procedure was performed in order to identify the statistical significance of the process factors. The results showed that all factors included in design had a significant impact on 
picking time. Taking into account all relevant goodness of model indices, all significant terms (with $p<0.05$ ) were included in the model, as shown in Tab. 1. The characteristics of the worker did not have a significant impact on picking time $(p>0.10)$.

\begin{tabular}{|c|c|c|c|c|c|c|}
\hline Source & $\begin{array}{l}\text { sum of } \\
\text { squares }\end{array}$ & $d f$ & $\begin{array}{l}\text { mean } \\
\text { square }\end{array}$ & $\begin{array}{c}F \\
\text { value }\end{array}$ & $\begin{array}{l}p \text {-value } \\
\text { prob }>F\end{array}$ & \\
\hline Model & 0.036728 & 10 & 0.00367 & 142.9 & $4.60 \mathrm{E}-130$ & sign. \\
\hline$A$ - Box size & 0.002625 & 2 & 0.00131 & 51.06 & $1.30 \mathrm{E}-20$ & \\
\hline$B$ - Box weight & 0.021562 & 1 & 0.02156 & 838.97 & $3.40 \mathrm{E}-103$ & \\
\hline$C$ - Shelf position & 0.000366 & 1 & 0.00037 & 14.22 & $1.90 \mathrm{E}-04$ & \\
\hline$A B$ & 0.000112 & 2 & 0.00006 & 2.18 & $1.10 \mathrm{E}-01$ & \\
\hline$B^{2}$ & 0.002033 & 1 & 0.00203 & 79.11 & $1.60 \mathrm{E}-17$ & \\
\hline$C^{2}$ & 0.001524 & 1 & 0.00152 & 59.3 & $9.40 \mathrm{E}-14$ & \\
\hline$A B^{2}$ & 0.000536 & 2 & 0.00027 & 10.43 & $3.80 \mathrm{E}-05$ & \\
\hline Residual & 0.011077 & 435 & 0.00003 & & & \\
\hline Lack of Fit & 0.000743 & 30 & 0.00002 & 0.96 & 5.30E-01 & not sign. \\
\hline Pure Error & 0.010334 & 401 & 0.00003 & & & \\
\hline Cor Total & 0.055191 & 445 & & & & \\
\hline
\end{tabular}

The main effects of the factors $(A, B$, and $C)$ can be seen in Fig. 2. Picking time is significantly impacted by the box weight. The difference in picking time of the $0.1 \mathrm{~kg}$ box and $10 \mathrm{~kg}$ box is about $1.7 \mathrm{sec}$ on average. Furthermore, the height of the platform where the box was positioned (shelf position) has a lower impact on the picking time. Therefore, when shelf position is 13 or 185 $\mathrm{cm}$ above the ground level in comparison to the nearoptimal $100 \mathrm{~cm}$, the difference is about 0.4 seconds.

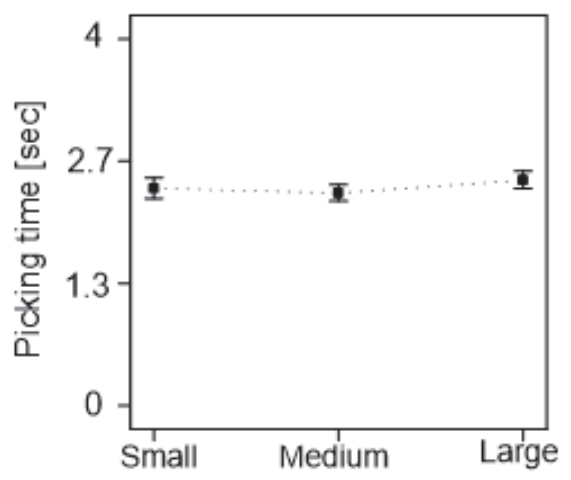

A: Box size

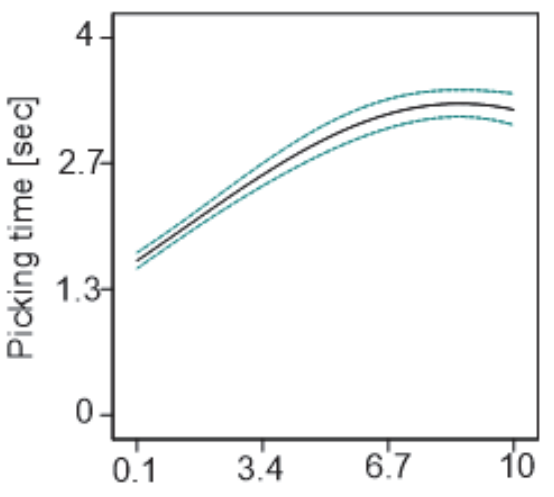

B: Box weight $[\mathrm{kg}]$

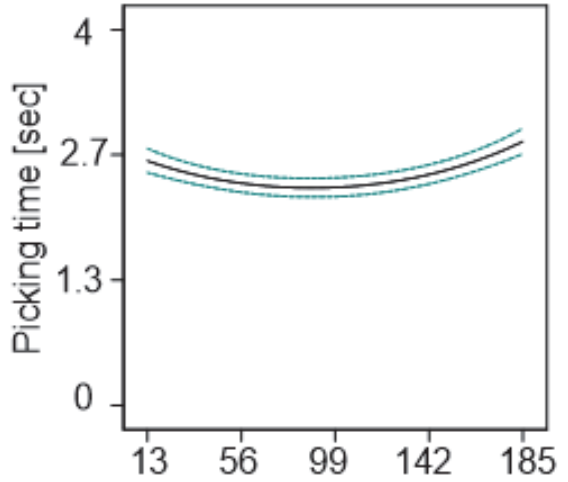

C: Shelf position $[\mathrm{cm}]$

Figure 2 The average effects of the factors with $95 \%$ confidence interval bands

Considering the factor $A$ (Box size), there is a small difference in picking time in comparison to the other two factors. However, it should also be included in the regression model.

The whole model includes higher order terms and interaction. The $R$-squared is about $77 \%$, which is a reasonable value taking into account that the residuals remain normally distributed. Due to the fact that the problem deals with two numerical factors and one categorical factor, the regression model for the prediction of picking time can be written in 3 separate equations. Eq. (2) refers to small, Eq. (3) to medium and Eq. (4) to large box size.

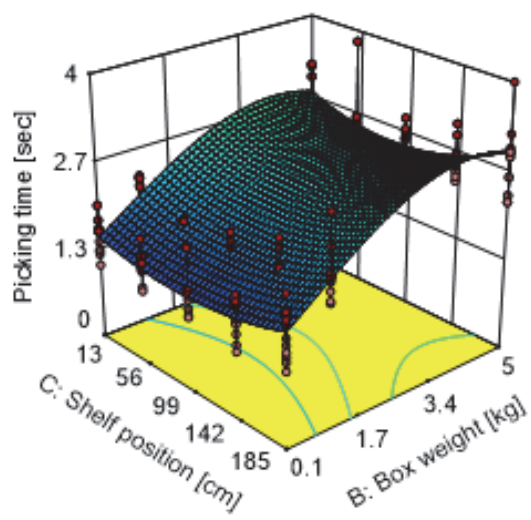

a)

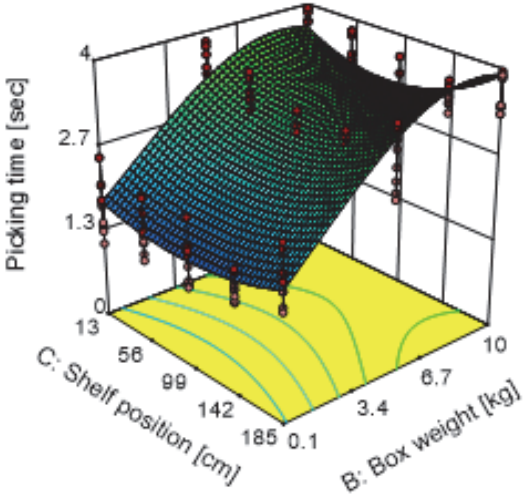

b)

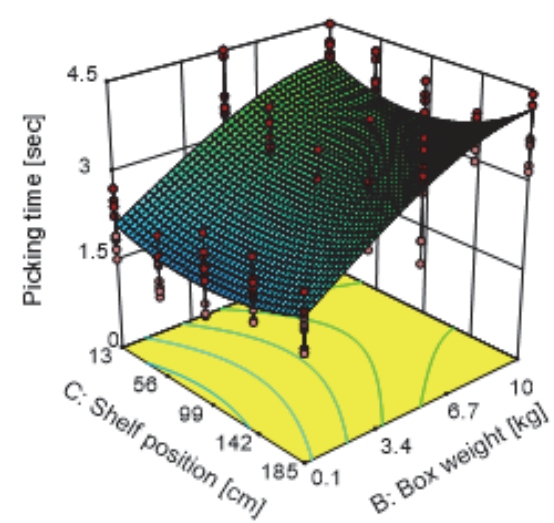

c)

Figure 3 Response surfaces for variable picking time a) Small box size; b) Medium box size; c) Large box size 
The response surface models constructed from given Eqs. (2), (3) and (4) are shown in Fig. 3.

$A=$ small box

$y^{0.03}=1.0137+9.7612 \times 10^{-3} * B-1.1275 \times 10^{-4} * C-$

$-1.2064 \times 10^{-3} * B^{2}+6.3908 \times 10^{-7} * C^{2}$

$A=$ medium box

$y^{0.03}=1.0189+5.15996 \times 10^{-3} * B-1.1275 \times 10^{-4} * C-$

$-3.0104 \times 10^{-4} * B^{2}+6.3908 \times 10^{-7} * C^{2}$

$A=$ large box

$y^{0.03}=1.02406+3.4835 \times 10^{-3} * B-1.1275 \times 10^{-4} * C-$

$-1.6346 \times 10^{-4} * B^{2}+6.3908 \times 10^{-7} * C^{2}$

\subsection{FILI Model}

To achieve the optimal solution in the bi-objective problem, we had to add to a picking time model also an ergonomic response surface. We experimented with the equation of the FILI index across the whole experimental area. We calculated the value of the FILI index for the experimental points with the Revised NIOSH Lifting Equation method. Experimental points were the same as those previously used for the calculation of the picking time model. In the next step, we researched the possibility of using the regression model of the FILI as an approximation. It is a challenge to simplify the calculations in multi-criteria experimental optimisation, which was performed in the specific computer algorithm (Design Expert 10).

The developed regression model with a high $R$-squared (98\%) is suitable for the prediction of the FILI index for all combinations of factors in the experimental area. When using $R N L E$, the only two values of factor $A$ - box size are different, small box and a larger one. Consequently, there are only two regression equations.

As shown in Fig. 4, it can be seen that the FILI index rises significantly in parallel with increases in the box weight. A minor but significant difference was noticed in FILI values on different shelf positions along the vertical of the warehouse rack.

$A=$ small box size

$$
\begin{aligned}
& y_{F I L I}^{0.5}=0.1199+0.1567 * B-1.8586 \times 10^{-3} * C- \\
& -8.0444 \times 10^{-3} * B^{2}+9.5953 \times 10^{-6} * C^{2}
\end{aligned}
$$

$A=$ medium and large box size

$$
\begin{aligned}
& y_{F I L I}^{0.5}=0.0605+0.1732 * B-1.8586 \times 10^{-3} * C- \\
& -8.0444 \times 10^{-3} * B^{2}+9.5953 \times 10^{-6} * C^{2}
\end{aligned}
$$

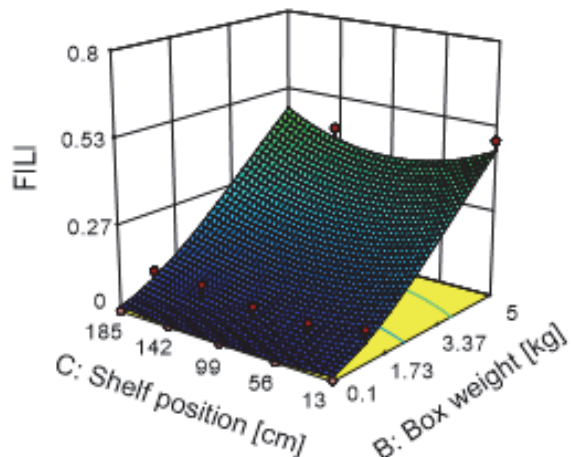

a)

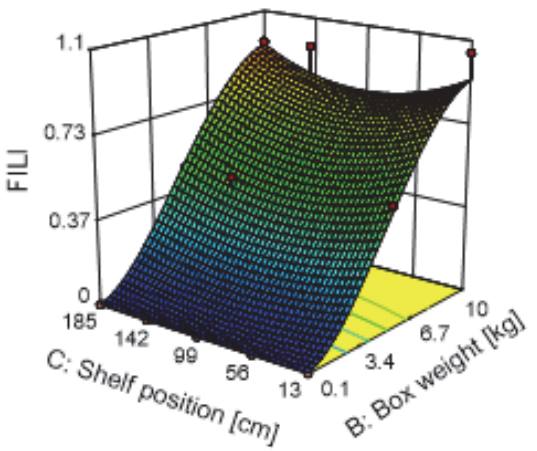

b)

Figure 4 Response surfaces of FILI model for a) small box sizes; b) large box sizes

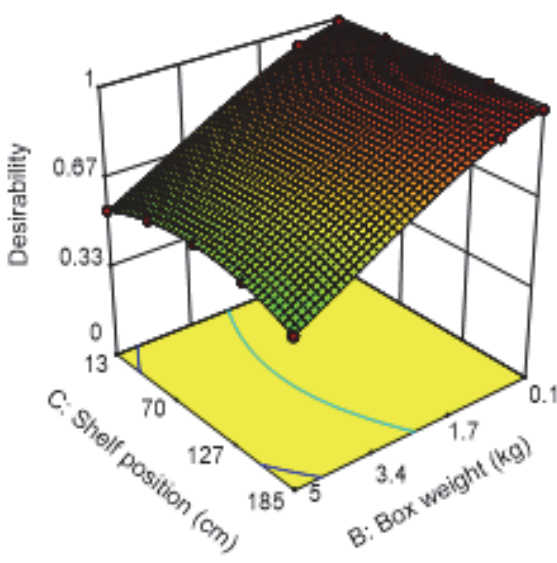

a)

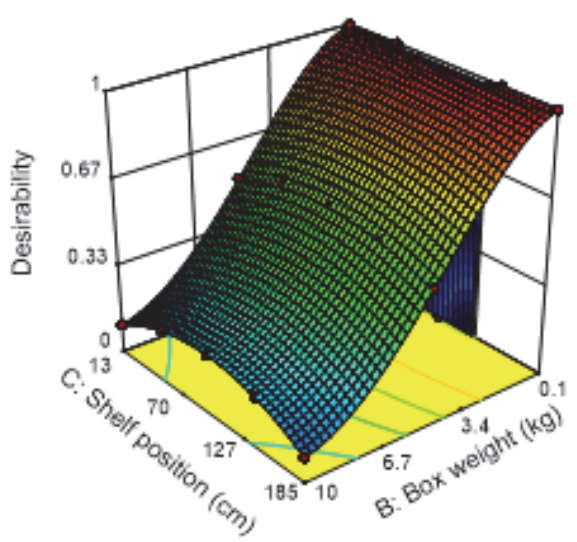

b)

Figure 5 Desirability function for the case a) small box size; b) large box size 
Examining the relationship between the picking time and FILI index, it can be concluded that they are highly positive significantly correlated with a coefficient of 0.81 . That proves the hypothesis of optimal solution at minimum values of FILI and picking time.

The optimal solution of bi-objective (multi-criteria) optimization was performed through experimental optimization using software package Design Expert 10. The result of the optimal solution is presented with constructed desirability function in Fig. 5. For the purpose of the demonstration of the optimal solution, the level of the importance of the criteria was set equally.

\section{DISCUSION AND CONCLUSIONS}

In the literature review, we did not find any paper addressing the storage assignment model, including optimisation in order picking time and structure of postures at order picking. Far from the forefront of warehouse management are efforts to improve productivity. Unfortunately, in parallel, we are recording increasing shares of WRMDs among employees, especially among manual workers, such as those in order picking processes. The occurrence of a specific posture can, if it occurs with a certain frequency, increase the likelihood of WRMDs occurring in the long run. When order picking, existing information systems do not adapt the set of tasks to employees. They are intended to classify work among order pickers treated as equal or standard in terms of physical strength, available energy for work and body height. Paper tests the possibility of bi-object treatment of the problem by adding to the time optimization the optimization of the frequency of occurrence of less or undesirable postures, which in the long run could affect the lower incidence of WRMDs at order-pickers.

Using a laboratory experiment, we determined retrieval times for units with different characteristics and studied associated body postures by guidelines of the Revised NIOSH Lifting Equation. We took a step closer to the bi-objective assignment model; time and risk for injuries.

In the laboratory experiment, we recorded picking times with the help of the time study method. Later these times were used for the development of a picking time model. Order pickers had to move boxes that differ in volume and mass. They were deposited on five different shelf positions in a vertical direction. All these factors have a significant impact on picking time. Among all impacts, the size of the box has the smallest impact on picking time. Workers who participated in the experiment were of different ages, weights, and heights. However, they all fit in the order picker profile. We wanted to get average picking times from the storage rack and not be satisfied with estimates based on subjective assumptions. This methodological approach is original, although a larger sample might be desired. We could not prove any significant impact of these characteristics on picking time. We were surprised that workers over the age of 50 were significantly slower in terms of picking large and heavy boxes above the head and at leg height. There were no significant statistical differences at picking boxes in the golden zone between younger and older order pickers [24].
To achieve the optimal solution in the bi-objective problem, we had to add to a picking time model also an ergonomic response surface. We experimented with the equation of the FILI index across the whole experimental area. We calculated the value of the FILI index for the experimental points with the Revised NIOSH Lifting Equation method. Experimental points were the same as those previously used for the calculation of the picking time model. In the next step, we researched the possibility of using the regression model of the FILI as an approximation. It is a challenge to simplify the calculations in multi-criteria experimental optimisation. From the theory of $R N L E$ we know, that if the FILI of the first task in order is less than 1.0, then this task is not stressful for the order picker in terms of strength requirements. Analysis of changes in FILI values ended with a finding that the FILI index rises significantly in parallel with increases in the box weight. A minor but significant difference was noticed in FILI values on different shelf positions along the vertical of the warehouse rack. At this stage of research experimentation, we report optimal solutions at minimum values of FILI and picking time. We evaluate the result as partial. The research must continue. FILI did not prove appropriate in the case of a multitasking process. Using FILI, we do not properly follow the contribution of additional lifting or lowering tasks. According to theory, FILI is only an indication of a specific task's stress for the order picker.

Manual picking is, in most cases, a multitasking job. Order pickers manually manipulate boxes of various masses and volumes stacked on different heights in the warehouse racks. We are satisfied with the developed picking time model. Still, to be able to solve a bi-objective problem, we need to find a solution for the improvement of the ergonomics model. The theory or RNLE proposes the method for analysing multitask processes. To continue the research, we will consider suitability to calculate the composite lifting index $C L I$ and build ergonomics model on this new basis.

\section{REFERENCES}

[1] See https://www.shmula.com/about-peter-abilla/what-ismura-muri-muda/

[2] De Koster, R., Le-Duc, T., \& Roodbergen, K. J. (2007). Design and control of warehouse order picking: a literature review. European Journal of Operational Research, 182(2), 481-501. https://doi.org/10.1016/j.ejor.2006.07.009

[3] Chan, F. T. S \& Chan, H. K. (2011). Improving the productivity of order picking of a manual-pick and multilevel rack distribution warehouse through the implementation of class-based storage. Expert Systems with Applications, 38, 2686-2700. https://doi.org/10.1016/j.eswa.2010.08.058

[4] Frazelle, E. A. (2002). World-class Warehousing and Material Handling. McGraw-Hill, New York.

[5] Tompkins, J. A., White, Y. A., Bozer, E. H., \& Tanchoco. J. M. A. (2010). Facilities Planning. Wiley, Hoboken, NY.

[6] Grosse, E. H., Glock, C. H., Jaber, M. J., \& Neumann, P. W. (2015). Incorporating human factors in order picking planning models: framework and research opportunities. International Journal of Production Research, 53(3), 695717. https://doi.org/10.1080/00207543.2014.919424 
[7] Gajšek, B., Đukić, G., Opetuk, T., \& Cajner, H. (2017). Human in manual order picking systems. MOTSP 2017: Conference proceedings.

[8] Harari, Y., Riemer, R., \& Bechar, A. (2018). Factors determining workers' pace while conducting continuous sequential lifting, carrying, and lowering tasks. Applied Ergonomics, 67, 61-70. https://doi.org/10.1016/j.apergo.2017.09.003

[9] Coenen, P., Kingma, I., Boot, C. R., Twisk, J. W., Bongers, P. M., \& van Dieen, J. H. (2013). Cumulative low back load at work as a risk factor of low back pain: a prospective cohort study. Journal of Occupational Rehabilitation, 23, 11-18. https://doi.org/10.1007/s10926-012-9375-z

[10] Manchikanti, L., Singh, V., Falco, F. J., Benyamin, R. M., \& Hirsch, J. A. (2014). Epidemiology of low back pain in adults. Neuromodulation Technology Neural Interface, 17, 3-10. https://doi.org/10.1111/ner.12018

[11] Punnett, L., Prüss-Ütün, A., Nelson, D. I., Fingerhut, M. A., Leigh, J., Tak, S., \& Phillips, S. (2005). Estimating the global burden of low back pain attributable to combined occupational exposures. American Journal of Industrial Medicine, 48, 459-469. https://doi.org/10.1002/ajim.20232

[12] Gallagher, S., Sesek, R. F., Schall Jr., M. C., \& Huangfu, R. (2017). Development and validation of an easy-to-use risk assessment tool for cumulative low back loading: The Lifting Fatigue Failure Tool (LiFFT). Applied Ergonomics, 63, 142-150. http://dx.doi.org/10.1016/j.apergo.2017.04.016

[13] Hoy, D., Bain, C., Williams, G., March, L., Brooks, P., Blyth, F., Woolf, A., Vos, T., \& Buchbinder, R. (2012). A systematic review of the global prevalence of low back pain. Arthritis Rheumatism, 64, 2028-2037. https://doi.org/10.1002/art.34347

[14] Calvo-Munoz, I., Gomez-Conesa, A., \& Sanchez-Meca, J. (2013). Prevalence of low back pain in children and adolescents: a meta-analysis. BMC Pediatrics, 13, 1. https://doi.org/10.1186/1471-2431-13-14

[15] Balague, F., Mannion, A.F., Pellise, F., \& Cedraschi, C. (2012). Non-specific low back pain. Lancet, 379, 482-491. https://doi.org/10.1016/S0140-6736(11)60610-7

[16] Sanders, A., Elangeswaran, C., \& Wulfsberg, J. (2016). Industry 4.0 implies lean manufacturing: Research activities in industry 4.0 function as enablers for lean manufacturing. Journal of Industrial Engineering and Management, 9, 811833. https://doi.org/10.3926/jiem.1940

[17] Grosse, E. H., Glock, C. H., \& Neumann, W. P. (2017). Human factors in order picking: A content analysis of the literature. International Journal of Production Research, 55(5), 1260-1276. https://doi.org/10.1080/00207543.2016.1186296

[18] Battini, D., Calzavara, M., Persona, A., \& Sgarbossa, F. (2015). A comparative analysis of different paperless picking systems. Industrial Management \& Data Systems, 115(3), 483-503. https://doi.org/10.1108/IMDS-10-2014-0314

[19] Price, A. D. (1990). Calculating relaxation allowances for construction operatives - Part 1: Metabolic cost. Applied ergonomics, 21(4), 311-317. https://doi.org/10.1016/0003-6870(90)90202-9

[20] Larco, J. A., de Koster, R., Roodbergen, K. J., \& Dul, J. (2016). Managing warehouse efficiency and worker discomfort through enhanced storage assignment decisions. International Journal of Production Research, 1-16. https://doi.org/10.1080/00207543.2016.1165880

[21] Borg, G. (1982). A Category Scale with Ratio Properties for Intermodal and Interindividual Comparisons. Psychophysical Judgment and the Process of Perception, 2534.

[22] Waters, T. R. (1994). Applications Manual for the Revised Niosh Lifting Equations. Cincinnati, Ohio: U.S. Department of Health and Human Services, Public Health Service.
[23] Temple, R. \& Adams, T. (2000). Ergonomic Analysis of a Multi-Task Industrial Lifting Station Using the NIOSH Method. Journal of Industrial Technology, 16(2), 1-6.

[24] Gajšek, B., Cajner, H., Đukić, G., Opetuk, T., Lukač, B., \& Puškadija, N. (2018). Ergonomics for productivity in orderpicking processes for aging workforce. Ergonomics 2018 Book of proceedings, 109-118.

[25] Okimoto, M. L. L. R. \& Teixeira, E. R. (2009). Proposed procedures for measuring the lifting task variables required by the Revised NIOSH Lifting Equation - A case study. International Journal of Industrial Ergonomics, 39, 15-22. https://doi.org/10.1016/j.ergon.2008.07.007

[26] Waters, T.R., Putz-Anderson, V., Garg, A., \& Fine, L. J. (1993). Revised NIOSH equation for the design and evaluation of manual lifting tasks. Ergonomics, 36(7), 74976. https://doi.org/10.1080/00140139308967940

[27] Colim A., Arezes P., Flores P., \& Braga A. C. (2019) Workers' Body Constitution as a Risk Factor During Manual Materials Handling. Advances in Intelligent Systems and Computing, 876. https://doi.org/10.1007/978-3-030-02053-8 136

[28] Chen, J. A., Dickerson, C. R., Wells, R. P., \& Laing, A. C. (2017). Older females in the workforce - the effects of age on psychophysical estimates of maximum acceptable lifting loads. Ergonomics, 60(12), 1708-1717. https://doi.org/10.1080/00140139.2017.1335883

\section{Contact information}

Brigita GAJŠEK, assist. prof., $\mathrm{PhD}$ (Corresponding author)

Faculty of logistics, University of Maribor,

Mariborska cesta 7, 3000 Celje, Slovenia

E-mail: brigita.gajsek@um.si

Hrvoje CAJNER, assist. prof., PhD

University of Zagreb,

Faculty of Mechanical Engineering and Naval Architecture

Ivana Lučića 5, Zagreb, Croatia

E-mail: hrvoje.cajner@fsb.hr

Marcin BUTLEWSKI, PhD

Poznan University of Technology,

Department of Ergonomics and Quality Management,

Poznań, Poland

E-mail: marcin.butlewski@put.poznan.pl

Tihomir OPETUK, assist. prof., PhD

University of Zagreb,

Faculty of Mechanical Engineering and Naval Architecture

Ivana Lučića 5, Zagreb, Croatia

E-mail: tihomir.opetuk@fsb.hr

Goran ĐUKIĆ, prof., PhD

University of Zagreb,

Faculty of Mechanical Engineering and Naval Architecture

Ivana Lučića 5, Zagreb, Croatia

E-mail: goran.dukic@fsb.hr 